\title{
Confocal microscopy of colloidal dispersions in shear flow using a counter-rotating cone-plate shear cell
}

\author{
Didi Derks, Hans Wisman, Alfons van Blaaderen and Arnout Imhof \\ Soft Condensed Matter, Debye Institute, Utrecht University, Princetonplein 5, 3584 CC Utrecht, \\ The Netherlands ${ }^{1}$ \\ E-mail: D.Derks@phys.uu.nl, A.vanBlaaderen@phys.uu.nl and A.Imhof@phys.uu.nl
}

Received 26 April 2004

Published 10 September 2004

Online at stacks.iop.org/JPhysCM/16/S3917

doi:10.1088/0953-8984/16/38/010

\begin{abstract}
We report on novel possibilities for studying colloidal suspensions in a steady shear field in real space. Fluorescence confocal microscopy is combined with the use of a counter-rotating cone-plate shear cell. This allows imaging of individual particles in the bulk of a sheared suspension in a stationary plane. Moreover, this plane of zero velocity can be moved in the velocity gradient direction while keeping the shear rate constant. The colloidal system under study consists of rhodamine labelled PMMA spheres in a nearly density and refractive index matched mixture of cyclohexylbromide and cis-decalin. We show measured flow profiles in both the fluid and the crystalline phase and find indications for shear banding in the case of a sheared crystal. Furthermore, we show that, thanks to the counter-rotating principle of the cone-plate shear cell, a layer of particles in the bulk of a sheared crystalline suspension can be imaged for a prolonged time, with the result that their positions can be tracked.
\end{abstract}

\section{Introduction}

The relation between microstructure and flow behaviour of soft condensed matter systems has been a subject of intensive research. Subtle changes in microstructure resulting from shear may lead to strong shear thinning or shear thickening behaviour. In many cases, however, shear may even lead to sudden and dramatic changes in the long range order of a complex fluid. Shear-induced structural transformations occur in colloidal dispersions, block copolymers and micellar solutions. Recent reviews can be found in [1-4]. Most information on shear-induced microstructures has been obtained with scattering measurements. It has become increasingly clear, however, that real space observations on the length scale of the individual particles are necessary for understanding these nonequilibrium phase transitions. Modern advanced microscopy techniques, such as confocal fluorescence microscopy, make it possible to study

1 www.colloid.nl 
the structure and dynamics of colloidal particles in three dimensions [5-7]. Extension of these methods to colloidal dispersions under shear is expected to lead to new insights into shear-induced phase transitions that have been investigated in the past and new phenomena that cannot be so easily inferred from a $k$-space analysis. The former include the formation of crystalline order under oscillatory shear [8], shear melting of colloidal crystals [9], shear banding in dispersions of spherical [10,11] and rod-like colloids [12].

Apart from the fundamental importance of understanding structural transformations in external shear flow, further motivation is found in the great importance of shear during industrial processing of colloidal systems such as foods, paints and emulsions. In addition, shear-induced large single-crystal formation can be used to prepare new advanced materials such as photonic crystals $[13,14]$.

In order to apply shear in a controlled way and to study the sheared system on a microscopic level a microscope needs to be equipped with more advanced tools than is necessary for the study of the equilibrium structure and dynamics of colloidal suspensions. With the development of shear cells that can be mounted on top of microscopes, investigations in real space became possible. Recently the effects of shear have been investigated in different types of set-up. Palberg et al [11] combined a plate-plate shear cell with video microscopy. The cover slip correction was adjusted such that the focusing depth was several times the interparticle spacing. This enabled them to study the distribution functions for the projections of particle positions into the flow-vorticity plane. The trajectories of individual particles could not be determined in this way. Tolpekin et al [15] used a rotating plate-plate cell in combination with confocal microscopy to investigate the formation and break-up of aggregates in colloidpolymer mixtures under shear. They used desmearing by image processing to correct for effects of particle motion by the flow. They had to stop the flow in order to study the structure of the aggregates in 3D. Similarly, Varadan et al [16] studied colloidal gels under shear by imaging immediately after stopping the flow. Quantitative data on individual particles during flow were obtained by Hoekstra et al for attractive particle systems as well as by Stancik et al for repulsive systems, because they limited their shear set-up to 2D [17, 18].

For a real space study of a 3D system under shear the main difficulty is that the particles move through the field of view of the microscope too rapidly, making it impossible to track them, except very close to the stationary wall where the flow velocity is small enough. Although the development of faster confocal scanning techniques, for example with the use of a spinning Nipkow disc [19], can mean a big step forward, the real answer to this problem lies in the use of a counter-rotation shear cell. In this approach the two parts of the cell rotate in opposite directions, such that a stationary plane is formed in the interior. Objects in this plane can thus be observed for extended periods of time. This concept was used already by Taylor to study droplet break-up during emulsion formation [20]. Recent implementations of the counter-rotation concept to study the behaviour of noncolloidal particles under shear are given in [21, 22]. However, these investigations were limited to low-magnification microscopy and dilute samples.

We have used a new counter-rotating cone-plate shear cell suitable for high-magnification confocal fluorescence microscopy to study the behaviour of concentrated dispersions of micrometre sized hard-sphere-like colloids under shear. By adjusting the ratio of angular velocities of the plate and cone the stationary plane can be placed anywhere in the bulk. This allows us to observe microstructure and particle dynamics in the bulk of the dispersion for extended periods of time.

This paper is organized as follows. First we introduce the counter-rotating shear cell in combination with the confocal microscope and describe the preparation of the colloidal dispersions in section 2. Then, in section 3, we demonstrate the instrument by measuring microscopic flow profiles in sheared colloidal fluids and particle dynamics in colloidal crystals. 


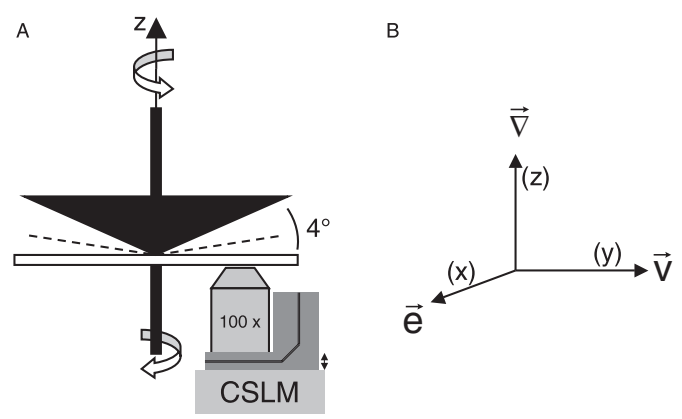

Figure 1. (A) A schematic representation of the experimental set-up. A cone-plate shear cell is placed on top of an inverted microscope. The metal cone and glass plate rotate in opposite directions. The dashed line indicates the zero-velocity plane. The objective is mounted on a piezodrive. In (B) the directions of the velocity gradient $\vec{\nabla}$, the flow $\vec{v}$ and the vorticity $\vec{e}$ are shown.

\section{Experimental section}

\subsection{Counter-rotating shear cell}

The measurements were performed with the Wageningen Centre of Food Science (WCFS) continuous shear cell configuration [23, 24]. This cone-plate shear cell was placed on top of an inverted microscope. A schematic representation is given in figure 1(A). The angle $\beta$ between cone and plate was $4^{\circ}\left( \pm 0.01^{\circ}\right)$. The cone and plate were driven independently by servomotors and rotated in opposite directions. The height of the stationary plane (zerovelocity plane) could be adjusted by tuning the ratio of the rotational speed of the cone and the plate, while the shear rate $\dot{\gamma}$ was kept constant. A key property of a cone-plate configuration is that the shear rate is the same at all radial positions: $\dot{\gamma}=\Delta \omega / \tan \beta$, where $\omega$ is the angular velocity and $\Delta \omega=\omega_{\text {cone }}-\omega_{\text {plate }}$.

In this set-up the radial position of the microscope objective was fixed. At this position the gap between the cone and plate was $1.70 \mathrm{~mm}( \pm 0.01 \mathrm{~mm})$. The cone was made of stainless steel. The plate consisted of a circular glass slide of diameter $8 \mathrm{~cm}$ and thickness $180 \mu \mathrm{m}$. The glass plate was clamped between two Teflon rings and supported in the centre by a metal disc. The inner diameter of the cell itself was $6.50 \mathrm{~cm}$. The cell was equipped with a vapour lock. Shear rates in a range of $10^{-2}-10^{2} \mathrm{~s}^{-1}$ could be applied.

During a full rotation we measured a variation in the gap width of $\pm 10 \mu \mathrm{m}$. This was mainly caused by a slight wobbling of the glass plate. The wobble in the cone was measured to be only $\pm 1 \mu \mathrm{m}$. This means that at typical shear rates the height of the glass plate, and thus the height of the stationary layer, fluctuates only very slowly. For example: at a shear rate of $5 \mathrm{~s}^{-1}$ and $\omega_{\text {cone }} / \omega_{\text {plate }}$ of 50 the height of the glass plate fluctuates by about $1.2 \mu \mathrm{m}$ over a time span of $1 \mathrm{~min}$. This is slow enough to allow adjustment of the height of the focal plane if necessary.

\subsection{Confocal microscopy and image analysis}

Particle imaging was performed using an inverted Leica confocal scanning laser microscope (CSLM), type TCS-SP2. The microscope was operated in the fluorescence mode. We used the $543 \mathrm{~nm}$ line of a green $\mathrm{He}-\mathrm{Ne}$ laser for excitation of the rhodamine labelled particles (and droplets). 3D imaging was made possible by the use of a piezo-focusing drive (Physik Instrumente P-721) of the objective lens. The scanning range of this drive was $100 \mu \mathrm{m}$. 
Measurements were performed with a $100 \times$ oil immersion objective with a numerical aperture of 1.4 and a working distance of $100 \mu \mathrm{m}$ (on top of the $180 \mu \mathrm{m}$ thick microscope slide). The microscope allows imaging of planes parallel as well as planes perpendicular to the glass plate. In the following the velocity gradient $\vec{\nabla}$ is directed along the $z$-axis; the flow $\vec{v}$ is directed along the $y$-axis and the $x$-axis points in the vorticity direction $\vec{e}$ (see figure 1(B)).

The coordinates of the colloidal particles were found in $x y$-layers using routines similar to those described by Grier and Crocker. In this way the particle coordinates could be obtained with sub-pixel accuracy. In our experiments the $x y$-pixel size is $73 \mathrm{~nm}$. The accuracy of finding the particle locations is estimated to be $10 \mathrm{~nm}$. The particles were tracked in time by comparing the coordinates found in every two successive frames from a time series of $x y$-images [25]. We used additional procedures to find velocity profiles in the shear field. Two routines were developed for measuring image drift due to the flow in time series of images taken parallel to the glass plate (perpendicular to the velocity gradient direction).

The first method uses particle coordinates and tracks as found above [25]. Then we consider every two successive frames and select all the particles that are present in both frames. Subsequently, we calculate the difference between the centres of mass of the selected particles in these two frames. Accumulation of these differences gives again the position shift as a function of time.

In the second method to measure drift we calculate the cross-correlation function of every two successive images. This function has a well-defined maximum at the value corresponding to the average displacement of all the particles. The position of this maximum is tracked to obtain the drift averaged over all particles in the frame as a function of time.

The two methods gave very similar results but were used for slightly different purposes: the first method for measuring the exact average velocity of the particles in the plane of zero velocity; and the second method for measuring the flow profile (from time series of different layers). Note that the second method has the advantage of being faster since no particle finding is necessary.

\subsection{Preparation of emulsions and colloidal suspensions}

Emulsions were prepared by making solutions of $10 \mathrm{wt} \%$ fish gelatine (Multi Product) and $10 \mathrm{wt} \%$ dextran (MW 282000) (Sigma) in water at $60{ }^{\circ} \mathrm{C}$, and mixing these in a ratio of $1 / 1$ at room temperature. In this way an emulsion of $10 \mu \mathrm{m}$ sized gelatine rich droplets in a continuous dextran rich phase was obtained. Rhodamine-B $(0.01 \mathrm{wt} \%)$ was added to the mixture which preferentially stains the gelatine rich phase $[24,26]$.

The colloidal particles used for this work were polymethylmethacrylate (PMMA) spheres prepared by dispersion polymerization [27]. The spheres were sterically stabilized by a graft copolymer of poly(12-hydroxystearic acid) (PHS) onto a PMMA backbone [27]. The reaction mixture initially consisted of the monomers methylmethacrylate (MM; Fluka) and methacrylic acid (MA; Fluka) dissolved in an apolar solvent: 2:1 (w/w) hexane (Aldrich)/Exxsol D 100 (Exxson Chemical Europe Inc.) mixture. The monomer concentration in the initial mixture was typically $40 \mathrm{wt} \%$. The particle size was tuned by adjusting the ratio (monomer)/(PHS stabilizer); a higher ratio resulted in larger particles. In the final locking step the stabilizer was chemically attached to the particles [27].

The particles were fluorescently labelled by adding a dyed monomer, which copolymerized with MM and MA. This fluorescent monomer was made beforehand by coupling rhodamineB-isothiocyanate (RITC, Aldrich) to amino-styrene (Aldrich), as described by Bosma et al [28]. In the present work several colloidal systems were used. The system labelled as D34 had a diameter $\sigma$ of $1.67 \mu \mathrm{m}$ and relative polydispersity $\xi$ of $5 \%$ (determined by static light 

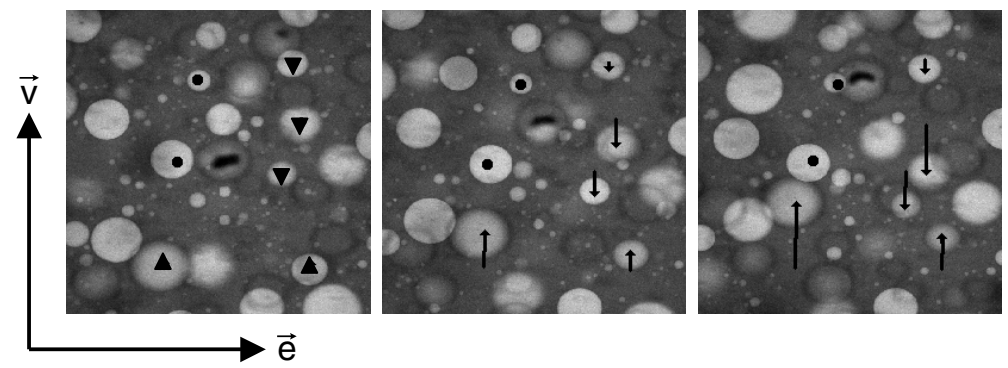

Figure 2. Confocal images $(x y, 75 \mu \mathrm{m} \times 75 \mu \mathrm{m})$ of a sheared gelatine-dextran emulsion (applied shear rate $\left.=1 \mathrm{~s}^{-1}\right)$. Snapshots were taken at time intervals of $10 \mathrm{~s}$. Stationary drops are marked with black circles; moving drops are marked with arrows pointing in the direction in which the drop moves.

scattering); D35: $\sigma=1.50 \mu \mathrm{m}$ and $\xi=6 \%$; DX22: $\sigma=1.32 \mu \mathrm{m}$ and $\xi=6 \%$. The last mentioned system consisted of core-shell cross-linked particles. Details about the synthesis of cross-linked particles with a fluorescent core and a non-fluorescent shell can be found in a recent paper by Dullens et al [29].

After the synthesis these systems were dried and redispersed in a 0.73:0.27 (w/w) mixture of cyclohexyl bromide (Fluka) and cis-decalin (Aldrich). In this mixture the mass density of the particles and the solvent were nearly matched, so the sedimentation or creaming of the particles was suppressed as much as possible. In addition, the refractive index of the particles was almost equal to that of the solvent (as well as to the refractive index of the glass plate of the shear cell and the immersion oil). The latter is important for imaging particles (in fluorescence mode) in bulk. In this solvent mixture the PMMA spheres were found to carry a charge [30, 31]. For these measurements the charges were screened by addition of an oilsoluble salt, i.e. tetrabutyl ammonium chloride (Sigma Aldrich) at a concentration of $260 \mu \mathrm{M}$. In this way the particles approximated hard spheres, crystallizing at a volume fraction of 0.47 .

\section{Results and discussion}

To illustrate the concept of the counter-rotating cell we first show measurements carried out on a gelatine-dextran emulsion. The droplet size distribution in the prepared macro-emulsion is very broad. A typical droplet size is about $10 \mu \mathrm{m}$, which means that its Brownian motion can be neglected. Successive confocal images of a sheared emulsion are shown in figure 2 . These $x y$-images were taken parallel to the glass plate and thus are perpendicular to the velocity gradient direction (=z-direction).

As a result of the applied shear field some droplets were seen to travel upward while others move downward. Some did not move at all, because their centre of mass was in the plane of zero velocity. For large objects such as these emulsion droplets we developed a computer controlled feedback mechanism that enabled tracking of an individual particle for a prolonged period of time. As soon as the selected droplet leaves the zero-velocity plane due to collisions with other droplets or due to slight mechanical drift, it starts drifting up or down with respect to the image plane. The computer then adjusts the ratio of the rotational speed of the cone and plate $\left(\omega_{\text {cone }} / \omega_{\text {plate }}\right)$ such that the drift is eliminated. The necessary angular velocities were calculated by determining the displacement of the droplet between two successive frames using image cross-correlation, under the constraint that the shear rate (and thus $\omega_{\text {cone }}-\omega_{\text {plate }}$ ) remained constant. In this way an emulsion droplet can be tracked indefinitely, so its deformation by the flow and by interactions with other droplets can be observed. 


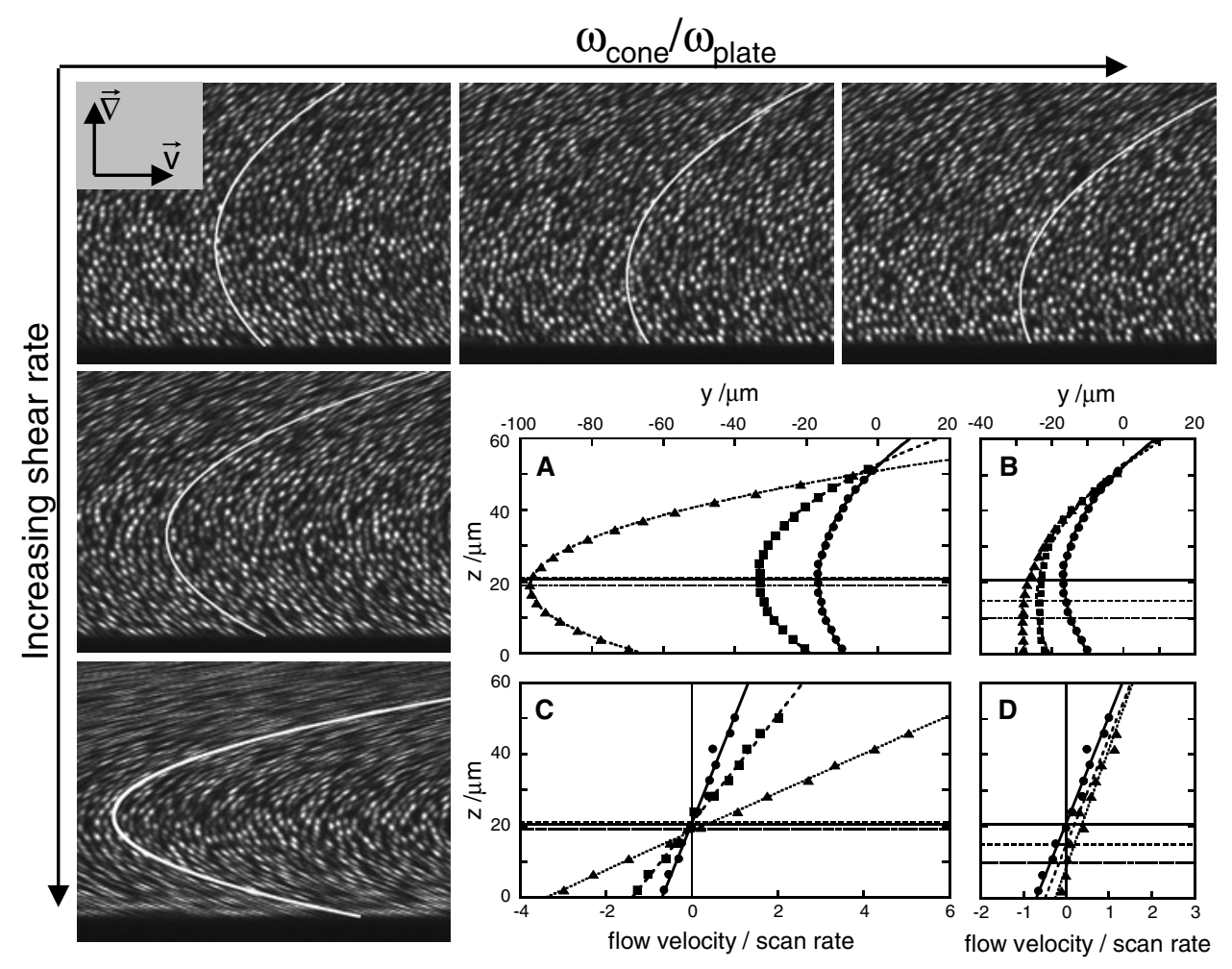

Figure 3. Confocal images ( $y z, 75 \mu \mathrm{m} \times 56 \mu \mathrm{m}, 512 \times 512$ pixels) of a colloidal fluid at various shear conditions. The applied shear rates are 1.67, 3.36 and $8.39 \mathrm{~s}^{-1}$ (top to bottom); the applied ratios $\left(\left|\omega_{\text {cone }} / \omega_{\text {plate }}\right|\right)$ are 84,129 and 175 (left to right). Images are overlaid (in white) with the calculated particle shape calculated from initial conditions following equation (2). Graphs A and B show the measured shape $(y(z))$ from these images resulting from image and data analysis (using the cross-correlation of lines). The velocity profiles obtained $(\mathrm{d} y / \mathrm{d} z)$ are shown in the graphs $\mathrm{C}$ and D. The particles used for this experiment have a diameter of $1.50 \mu \mathrm{m}$ (D35).

Unlike in a sheared macro-emulsion, in a sheared colloidal suspension all the particles in focus travel at nearly the same velocity. The reason is that the centres of mass of all the imaged particles lie at the same depth to within about $1 \mu \mathrm{m}$ (the diameter). Another essential difference from the emulsion droplets is that the colloidal particles perform Brownian motion. In colloidal dispersions the particles move in and out of the zero-velocity plane too rapidly for them to be tracked at the frame rates that we are using (maximum 4 frames s ${ }^{-1}$ for $256 \times 256$ pixels). Colloidal crystals represent an exception to this, because here the particles are confined to their lattice positions. We will demonstrate this later. In colloidal fluids we can gain most information on the flow profiles by imaging parallel to the velocity gradient ( $=z$-direction) and the flow direction (=y-direction). Scanning frames in such a way results in images in which the particles seem to be deformed into slanted ellipses (see figure 3 ). The deformation originates from the fact that scanning an image takes time. The image is scanned line by line from bottom to top: $z(t)=\alpha t$, with $\alpha$ the scan rate; thus the top and bottom will be displaced relative to each other by an amount $y_{\text {top }}-y_{\text {bottom }}=v(z) \Delta t=v(z)\left(z_{\text {top }}-z_{\text {bottom }}\right) / \alpha$, where $v(z)$ is the velocity of a particle of diameter $\sigma$ of which the centre of mass is situated at height $z$ in the shear field. This equation can be rewritten as

$$
\frac{y_{\text {top }}-y_{\text {bottom }}}{\sigma}=\frac{v(z)}{\alpha} \text {. }
$$




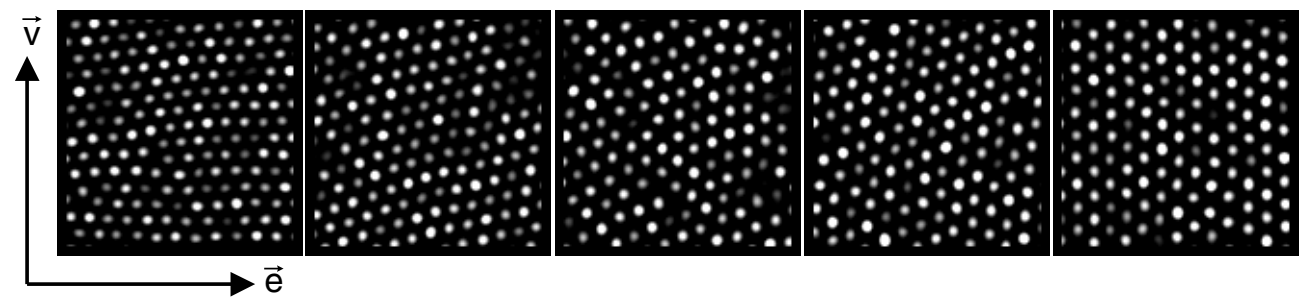

Figure 4. Reorientation of the hexagonal layer after applying a shear field. The applied shear rate yields $0.167 \mathrm{~s}^{-1}$. Snapshots are taken at $t=0,65,130,195$ and $390 \mathrm{~s}$; the shear is initiated at $t \cong 5 \mathrm{~s}$. Images are $37.5 \mu \mathrm{m} \times 37.5 \mu \mathrm{m}(128 \times 128$ pixels $)$. The particles used here are core-shell particles (DX22) with a diameter of $1.32 \mu \mathrm{m}$.

The latter expression equals $\mathrm{d} y / \mathrm{d} z$ and describes the inclination of the imaged particles as a function of the height. This slope can be determined by analysing the confocal images. In order to do so we first aim to find the apparent shape of the imaged particles $y(z)$. We wrote a routine that calculated the cross-correlation function of every two successively scanned lines. In the next step the positions of the maxima of these cross-correlation functions were tracked and accumulated, resulting in the final shape $y(z)$.

The results of this analysis are shown in the two upper graphs in figure 3. Graph A shows the curves obtained for experiments at three different shear rates corresponding to the images on the left side. Graph B shows the apparent shape for experiments done at different ratios $\omega_{\text {cone }} / \omega_{\text {plate }}$ (and constant $\omega_{\text {cone }}-\omega_{\text {plate }}$ ) corresponding to the images displayed at the top. The derivatives $\mathrm{d} y / \mathrm{d} z$ of these curves directly yield the flow profile (equation (1)). These flow profiles are plotted in graphs $\mathrm{C}$ and $\mathrm{D}$. It can be seen that increasing the shear rate while keeping the ratio $\omega_{\text {cone }} / \omega_{\text {plate }}$ fixed keeps the zero-velocity plane at the same height to within $2 \mu \mathrm{m}(\mathrm{C})$. On the other hand, increasing the ratio $\omega_{\text {cone }} / \omega_{\text {plate }}$ lowers the stationary layer while the shear rate stays constant (D).

When we deal with a simple shear flow the flow profile is given by $v(z)=\dot{\gamma}\left(z-z_{z v p}\right)$, where $z_{z v p}=\frac{h}{\left|\omega_{\text {cone }} / \omega_{\text {plate }}\right|+1}$. In our case, the gap $h$ is $1.70 \mathrm{~mm}$. The expression for the deformation now becomes parabolic:

$$
y(z)=\frac{\dot{\gamma}}{\alpha} z\left(\frac{1}{2} z-z_{z v p}\right)+\text { const. }
$$

Hence, for a linear velocity profile over the whole gap, the apparent shape of the imaged particles is completely determined by the applied shear rate and the applied ratio $\omega_{\text {cone }} / \omega_{\text {plate }}$. In figure 3 these calculated curves are plotted over the confocal images. The calculated shape agrees very well with the images, which means that the measured velocity profiles are indeed linear. For the data in figure 3 the scan rate $\alpha$ is $34 \mu \mathrm{m} / \mathrm{s}$. Therefore it takes about $40 \mathrm{~ms}$ to scan a particle from top to bottom. The Brownian motion during this time period is small enough not to disturb the analysis: the root mean square displacement is about $100 \mathrm{~nm}(=\sigma / 15)$, assuming infinite dilution, and will even be significantly less because of particle interactions and hydrodynamics.

In addition to fluids, it is also interesting to study colloidal crystals in shear flow. The first observation when applying a shear field to a crystal consisting of hexagonal stacked layers is that the layers reorient themselves in the steady shear field. Regardless of the original crystal orientation, the applied shear orients the hexagonal planes in such a way that close-packed lines point into the flow direction. The process of reorientation is demonstrated in figure 4 . 

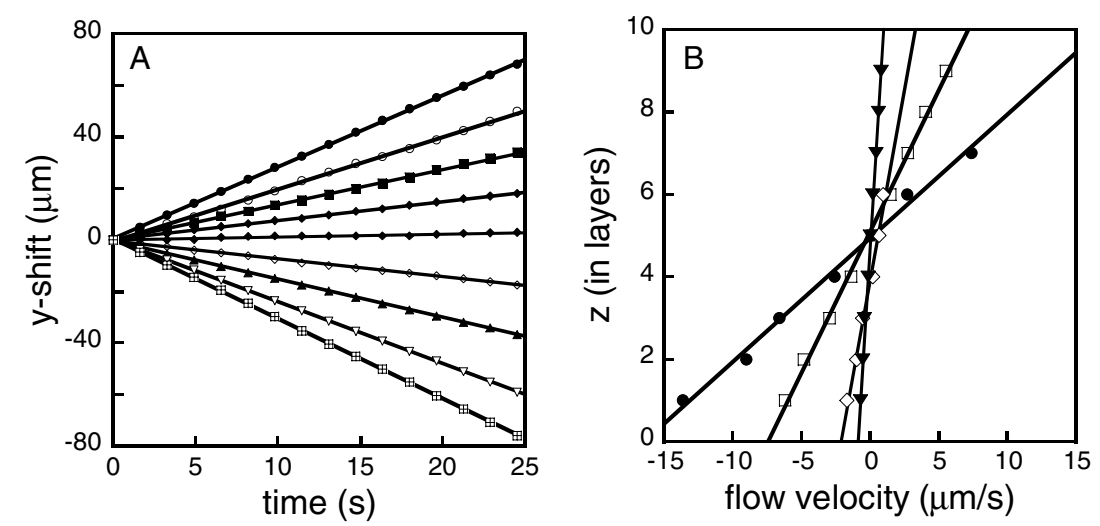

Figure 5. The velocity profile in the sheared crystal is measured layer by layer. (A) Image analysis of time series of $x y$-images results in the $y$-position shift as a function of time. ( $\boxplus$ ) corresponds to the layer closest to the glass plate; $(\mathbf{O})$ corresponds to the ninth layer (counted from the glass wall). (B) Slopes from (A) give the layer velocity as a function of height. Data from (A) correspond to $(\square)$ in (B). The measured shear rates obtained are $0.19 \mu \mathrm{m} \mathrm{s}^{-1} /$ layer $(\boldsymbol{\nabla}), 0.54 \mu \mathrm{m} \mathrm{s}^{-1} /$ layer $(\diamond)$, $1.46 \mu \mathrm{m} \mathrm{s}^{-1} /$ layer $(\square)$ and $3.33 \mu \mathrm{m} \mathrm{s}^{-1} /$ layer $(\bigcirc)$. Assuming a layer spacing of $\sqrt{2 / 3}$ times the measured interparticle spacing $d$ in the $x y$-plane $(d=1.80 \mu \mathrm{m}$; layer spacing $=1.47 \mu \mathrm{m})$ these numbers correspond to actual shear rates of $0.13,0.37,1.0$ and $2.3 \mathrm{~s}^{-1}$. For these experiments we used particles with a diameter of $1.67 \mu \mathrm{m}$ (D34).

In a colloidal crystal most of the particles are confined to lattice positions, so they can be tracked in the plane of zero velocity for a long period of time. To stay away from the shear melting transition the applied shear rates here had to be an order of magnitude lower than the shear rates that we applied to the colloidal fluid.

The advantage of these low shear rates is that the particles can be imaged not only in the plane of zero velocity, but also in a wider $z$-range around this plane. In fact, time series of $x y$-confocal images were captured in about ten layers around the zero-velocity plane. For each layer we used cross-correlation of successive images to calculate the motion due to flow (see experimental section). A typical example is shown in figure 5(A). The slopes of the lines correspond to the velocities of these layers. For experiments done at four different shear rates these velocities are plotted in figure 5(B) as a function of height (expressed in terms of number of layers from the glass plate). The slopes of the lines in this graph give the actual shear rates in the four experiments. From figure 5(A) it can be seen that every layer moves with a velocity that is intermediate between the velocities of its two neighbouring layers. In other words: all layers slide over each other; no two layers ever move in unison. We found this in all experiments and therefore we conclude that in the lower $100 \mu \mathrm{m}$, where we could image, the flow profile in colloidal crystals is linear down to the particle level.

On comparing the measured shear rates with the applied ones we found, as expected from figure 3, good agreement for the colloidal fluid samples (figure 6(A)). However, in the case of the sheared crystal, we observed a remarkable difference. The measured shear rates are much higher than those applied (figure 6(B)). The discrepancy cannot be explained by wall slip because this would lead to measured shear rates that are too low. We conclude that, although we have no direct evidence yet, the velocity profile cannot be linear throughout the whole gap. We believe that this is an indication of shear banding.

The measurements shown here were performed with an objective with a free working distance of $100 \mu \mathrm{m}$. This did not allow us to image particles throughout the whole gap, which 

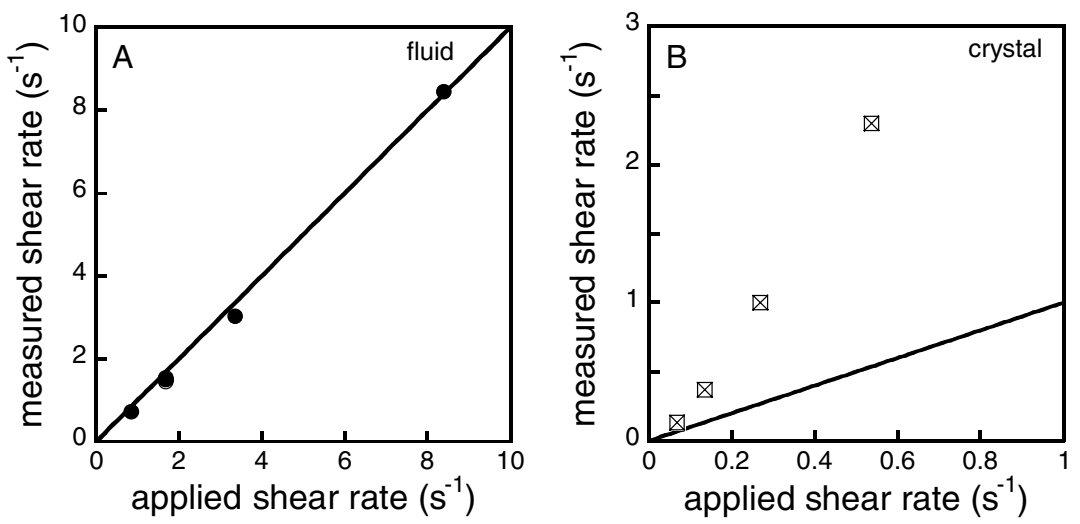

Figure 6. Measured shear rate versus applied shear rate for the sheared fluid (A) and crystal (B). Solid lines represent: measured shear rate = applied shear rate. Plotting the measured shear rate as a function of the applied shear rate demonstrates an interesting discrepancy from the applied shear rate in the case of the crystal, while the fluid shows normal behaviour. For the crystal the shear rate in this region is much higher than expected.

is $1.7 \mathrm{~mm}$. Future work will involve the use of longer working distance objectives so that information can be gained over a larger distance in the gap.

In the following we discuss results concerning particle dynamics in the plane of zero velocity of a sheared crystal. The combination of a counter-rotating shear cell and a confocal microscope gives the opportunity to image one stationary layer exclusively in a 3D bulk system under shear. In this way we are able to analyse the dynamics of the colloidal particles in real space in great detail.

At moderate shear rates (up to several $\mathrm{s}^{-1}$ ) the particles in an ordered layer in the zerovelocity plane can stay in focus for a sufficiently long time for one to measure individual particle trajectories. The higher the shear rate the more difficult it is to keep the particles in focus, for several reasons. Firstly, the glass plate is moving at higher speed and as a result more variations in height are encountered during the time of the experiment. Secondly, the height of the crystalline plane is fluctuating more. Thirdly, rearrangements in the layer, a precursor to shear melting, become more frequent.

To study the particle dynamics in the zero-velocity plane, time series (typically 256 frames) were captured over a region of $18.75 \mu \mathrm{m} \times 18.75 \mu \mathrm{m}(256 \times 256$ pixels $)$ with a high scan rate $\left(3.9\right.$ frames $\left.^{-1}\right)$. Figure $7(\mathrm{~A})$ shows a representative image from such a series. Image analysis gave the coordinates of the particles in the successive frames. Subsequently, these coordinates were ascribed to the individual particles, producing all the trajectories. Directly plotting these tracks gives a tangle of curves from which not much can be learned. After subtracting the collective motion obtained by averaging over all the particles we end up with spatially separate tracks, from which we observe particles moving around their average lattice positions (figure 7(B)).

The average collective motion of the frames is obtained by calculating the shift in the position of the centre of mass between two successive frames, weighting all the particles that appear in both frames. This collective motion does give us information on how the layer as a whole moves in the applied shear flow. As we learned from the measurements of flow profiles in a crystal, all the layers do slide over each other. So, if we want to learn how the particles behave when the layers slide over each other, we should also study the collective motion in more detail [32]. 

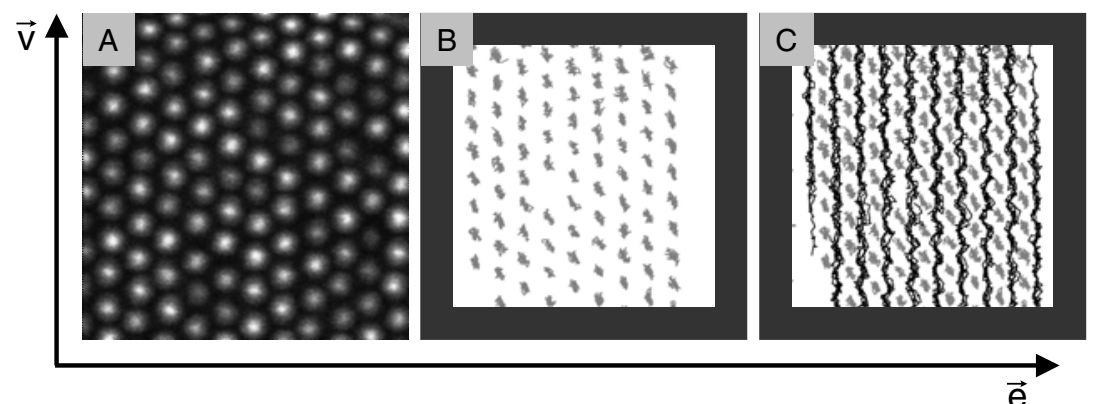

Figure 7. Image analysis and particle tracking in the zero-velocity plane reveal the dynamics of the sheared crystal. Moreover, dynamical information from two layers at the same time is gained. The zigzag motion of the particles from one layer over the adjacent layer is clear for the image at the right. The image size is $18.75 \mu \mathrm{m} \times 18.75 \mu \mathrm{m}(256 \times 256$ pixels $)$. The colloids used have a diameter of $1.50 \mu \mathrm{m}$ (D35).

Of course it would be most informative if the positions of particles in several layers could be measured simultaneously, and correlated. This was approximated by taking time series as before, but now alternately scanning one layer $\left(z_{\text {layer1 }}\right)$ and the layer above $\left(z_{\text {layer2 }}\right)$. Note that there are some differences with the $2 \mathrm{D}$ time series described above. One difference is that strictly speaking only one layer can be in the zero-velocity plane, while in the other layer the particles will move a bit more. Another difference is that the time interval between two images of the same layer is longer than for a 2D time series. Both effects reduce the maximal shear rate that can still be applied in order to obtain analysable data.

In figure 7(C) we plot the tracks of both layers. Here the small residual drift of the stationary layer was subtracted from the particles in both layers, producing their relative motions only. In this way we see the particles in the adjacent layer slide over (and move through the holes in between) the particles in the stationary layer. From this plot it can be qualitatively seen that the particles perform a zigzag motion when the layers are sliding over each other, as was already inferred by Ackerson et al from light scattering data [33]. A more detailed analysis of this zigzag motion will be published elsewhere [32].

\section{Conclusions}

A counter-rotating shear cell combined with confocal microscopy opens many possibilities for studying colloidal systems, as well as larger non-Brownian objects, in a shear flow in real space quantitatively on a single-particle basis. Flow profiles can be measured on a microscopic level in colloidal fluids and crystals. Strong indications for shear banding in sheared hardsphere-like crystals were found. Particles in the zero-velocity plane of a sheared colloidal crystal can be tracked. Finally, we have shown the possibility of capturing and tracking two layers (almost) simultaneously: as an example we demonstrated the zigzag motion with which close-packed planes of hard spheres slide over each other.

\section{Acknowledgments}

The continuous shear cell was designed in close collaboration with the Wageningen Centre for Food Science (WCFS), Forschungszentrum Jülich (FZ-J), the University of Twente and Unilever Research, from which we would especially like to thank Yves Nicolas, Marcel 
Paques (WCFS) and Jan Dhont (FZ-J). We thank Paddy Royall for critically reading the manuscript. This work is part of the research programme of the Stichting voor Fundamenteel Onderzoek der Materie (FOM), which is financially supported by the Nederlandse Organisatie voor Wetenschappelijk Onderzoek (NWO).

\section{References}

[1] Butler P 1999 Curr. Opin. Colloid Interface Sci. 4214

[2] Hamley I W 2000 Curr. Opin. Colloid Interface Sci. 5342

[3] Richtering W 2001 Curr. Opin. Colloid Interface Sci. 6446

[4] van Blaaderen A 2004 Mater. Res. Soc. Bull. 2985

[5] van Blaaderen A 1997 Prog. Colloid Polym. Sci. 10459

[6] Kegel W K and van Blaaderen A 2000 Science 287290

[7] Gasser U, Weeks E R, Schofield A, Pusey P N and Weitz D A 2001 Science 292258

[8] Pusey P N, van Megen W, Bartlett P, Ackerson B J, Rarity J G and Underwood S M 1989 Phys. Rev. Lett. 63 2753

[9] Ackerson B J and Clark N A 1981 Phys. Rev. Lett. 46123

[10] Imhof A, van Blaaderen A and Dhont J K G 1994 Langmuir 103477

[11] Palberg T and Biehl R 2003 Faraday Discuss. 123133

[12] Dhont J K G, Lettinga M P, Dogic Z, Lenstra T A J, Wang H, Rathgeber S, Carletto P, Willner L, Frielinghaus H and Lindner P 2003 Faraday Discuss. 123157

[13] Amos R M, Rarity J G, Tapster P R, Shepherd T J and Kitson S C 2000 Phys. Rev. E 612929

[14] Sawada T, Suzuki Y, Toyotama A and Iyi N 2001 Japan. J. Appl. Phys. 240 L1226

[15] Tolpekin V A, Duits M H G, van den Ende D and Mellema J 2004 Langmuir 202614

[16] Varadan P and Solomon M J 2003 J. Rheol. 47943

[17] Hoekstra H, Vermant J and Mewis J 2003 Langmuir 199134

[18] Stancik E J, Gavranovic G T, Widenbrant M J O, Laschitsch A T, Vermant J and Fuller G G 2003 Faraday Discuss. 123145

[19] Tanaami T, Otsuki S, Tomosada N, Kosugi Y, Shimizu M and Ishida H 2002 Appl. Opt. 414704

[20] Taylor G I 1934 Proc. R. Soc. 146501

[21] Grizzuti N and Bifulco O 1997 Rheol. Acta 36406

[22] Breedveld V, van den Ende D, Bosscher M, Jongschaap R J J and Mellema J 2001 Phys. Rev. E 63021403

[23] Paques M, Imhof A, van Blaaderen A and Nicolas Y 2001 European Patent Application 1312910

[24] Nicolas Y, Paques M, van den Ende D, Dhont J K G, van Polanen R C, Knaebel A, Steyer A, Munch J P, Blijdenstein T B J and van Aken G A 2003 Food Hydrocolloids 17907

[25] Crocker J C and Grier D G 1996 J. Colloid Interface Sci. 179298

[26] Nicolas Y, Paques M, Knaebel A, Steyer A, Munch J P, Blijdenstein T B J and van Aken G A 2003 Rev. Sci. Instrum. $\mathbf{7 4} 3838$

[27] Antl L et al 1986 Colloid Surf. 1767

[28] Bosma G, Pathmamanoharan C, de Hoog E H A, Kegel W K, van Blaaderen A and Lekkerkerker H N W 2002 J. Colloid Interface Sci. $\mathbf{2 4 5} 292$

[29] Dullens R P A, Claesson M, Derks D, van Blaaderen A and Kegel W K 2003 Langmuir 195963

[30] Yethiraj A and van Blaaderen A 2003 Nature 421513

[31] Royall C P, Leunissen M E and van Blaaderen A 2003 J. Phys.: Condens. Matter 15 S3581

[32] Derks D et al 2004 in preparation

[33] Ackerson B J 1990 J. Rheol. 34553 and references there in 